Check for updates

Cite this: RSC Adv., 2019, 9, 7469

Received 18th January 2019

Accepted 18th February 2019

DOI: 10.1039/c9ra00453j

rsc.li/rsc-advances

\section{Tailor-made synthesis of an melamine-based aminal hydrophobic polymer for selective adsorption of toxic organic pollutants: an initiative towards wastewater purification $\dagger$}

\author{
Debanjan Dey, ${ }^{\text {ab }}$ Naresh Chandra Murmu ${ }^{\text {ab }}$ and Priyabrata Banerjee (DD *ab
}

A cost-effective melamine-based polyaminal covalent polymer (CPCMERI-2) has been prepared by a facile synthetic approach using the solvothermal condensation reaction and characterized by solid-state analytical tools like ${ }^{13} \mathrm{C}$ NMR, PXRD, $\mathrm{N}_{2}$ sorption isotherm and FT-IR. The electron-rich moieties in the skeletal backbone induce hydrophobicity in the polymer with an appreciable water contact angle of $130^{\circ}$. AFM study establishes the plausible reason for the hydrophobicity. On account of its high thermal and chemical stability, the polymer CPCMERI-2 has been projected as a next-generation sorbent material for oil-like materials, and executed liquid-phase adsorption of kerosene over water surface. CPCMERI-2 selectively adsorbs kerosene and has a feeble adsorption affinity towards diesel and some other organic solvents like chloroform, benzene, nitrobenzene, and toluene. To improve the biocompatibility and cost effectiveness of the material, a bio-waste material like the peel of Citrus limetta is used in the composite material, and it unveils a new avenue towards exploring the use of naturally abundant bio-material peels as low-cost sorbent materials. Additionally, CPCMERI-2 has gained attention due to its enormous potential in wastewater purification, which has also been tested in a labscale experimental setup. We expect that this material (CPCMERI-2) will harbinger a new type of composite polymer, wherein naturally abundant waste bio-materials could be used as precursors to explore its usefulness as an adsorbent for the removal of oils and organic pollutants.

\section{Introduction}

The Schiff base condensation reaction has been a classical approach since its discovery in the year 1864 lead by Hugo Schiff, and it is considered to be a unique approach due to its versatility in organic synthesis. ${ }^{1-3}$ In such sort of reactions, the designed development of condensation products leading to imine bond formation is immensely helpful in constructing multi-dimensional functional materials. ${ }^{4-6}$ Furthermore, the developed imine bonds could be attacked by primary amines in proper experimental conditions resulting in an unprecedented generation of aminal linkages. ${ }^{7}$ The designed development of such polycondensation mediated materials by employing a one-

${ }^{a}$ Surface Engineering and Tribology, Central Mechanical Engineering Research Institute, Mahatma Gandhi Avenue, Burdwan District, Durgapur 713209, West Bengal, India. E-mail: pr_banerjee@cmeri.res.in; priyabrata_banerjee@yahoo.co.in; Web: www.priyabratabanerjee.in

${ }^{b}$ Academy of Scientific and Innovative Research, Anusandhan Bhawan, 2 Rafi Marg, New Delhi 110001, India

$\dagger$ Electronic supplementary information (ESI) available: TGA of CPCMERI-2, BJH pore size distribution, $\mathrm{CO}_{2}$ adsorption isotherm of CPCMERI-2 before and after kerosene uptake and FT-IR spectrum of CPCMERI-2 after four times recycling study of kerosene uptake. See DOI: 10.1039/c9ra00453j pot synthetic procedure is to the best of our knowledge hitherto less explored in the domain of functional covalent organic polymer (COP) synthesis. COPs are a class of materials in which there is less probability of leaching of any metal ion, because the entire polymer consists of only organic entities and the building units are stitched together by strong covalent bonding. ${ }^{8}$ Repeated trials and errors resulted in the successful development of a highly cross-linked mesoporous covalent organic polymer, ultimately showing its functional properties owing to its widespread three dimensional polymeric networks along with the rapid conversion of the initially generated imine functional group to an aminal one. The unique covalent linkages and the structural framework of the polymer are the chief components responsible for oil spill cleanup. ${ }^{9}$ As oil is nonpolar in nature there is a probability of better interaction with non-polar materials like COPs. Herein, the stacked electron-rich aromatic rings interact with the hydrophobic oil materials. Moreover, such COP materials are composed of very lightweight atoms like carbon and nitrogen in the aromatic precursors, and as a consequence the density of these materials are very low leading to their easy floatability over water surface ultimately helping in addressing burning issues like cleanup of spilled oil. The covalent bonds existing in the COPs are very 
strong and high energy is required to break these linkages, which account for their robust nature. ${ }^{10-13}$ As the COPs are entirely made up of organic units, the presence of electron-rich aromatic rings will assist in the staking of the polymer, which in turn will make the polymer nonpolar as well as hydrophobic.

In this connection, it is noteworthy that there are several naturally abundant bio-materials in nature which are easily accessible; however, to date, their implementation is explored inappreciably. Sometimes these so-called waste materials are used for the purpose of vermicomposting. Apart from that they can also be exploited in the form of adsorbents for the development of different functional materials. In this context, the proper use of such non-polar bio-waste materials with rich aromatic moieties makes them potential candidates in the development of composite COP materials for spilled oil cleanup. ${ }^{\mathbf{1 4 1 5}}$ They perhaps can be highlighted as promising, proficient, potential and cost-effective COP materials for oil spill cleanup and other purposes too.

In the $21^{\text {st }}$ century, various fuel oils serve essential purposes in our daily life. Petroleum products like fuel oil, kerosene and diesel are generally used for transportation and illumination purposes. These also have major uses in engineering products, automobile sectors etc. Fossil fuels like petroleum-related natural oils are becoming precious day by day, owing to the nonrenewable nature of these energy resources. However, till date, there have been several unfortunate incidents where fossil fuels have been accidentally spilled over sea and aquatic systems, causing threat to human as well as aquatic life, and in turn making this wealth hazardous from environmental perspective. ${ }^{\mathbf{1 6 - 2 0}}$ Generally, PAH in fossil fuels is exposed to the water bodies through spillage of oils by accidents (from several industries, oil refining plants, offshore oil spills, leakage from oil tankers during transportation etc.), from industries, or in the form of by-products coming from private or commercial resources. ${ }^{21}$ Light oils like kerosene during spillage hamper the dissolved oxygen level in the floating water bodies and ultimately have adverse impacts on the aquatic ecosystem. Moreover, the toxic constituents of oil particles in turn decrease the fertility of the land resulting in the intoxication of groundwater by leaching through contaminated soil. ${ }^{22}$ Therefore, such unwanted incidents necessitate the urge for the development of proper adsorbent materials which can remove oil from the surface of water bodies at a bare minimum cost. Literature reveals that a few organic polymeric materials like magnetic carbon-metal composite, $^{23}$ modified sponge, ${ }^{24-26}$ porous polymeric monoliths, ${ }^{27,28}$ covalent porphyrin framework, ${ }^{29}$ boron nanosheets, ${ }^{30,31}$ conjugated microporous polymers, ${ }^{32}$ and organic copolymers ${ }^{9}$ are used for oil spill clean-up purpose. However, their multistep formation route, use of costly precursor materials, tricky reaction conditions etc. hinder their application for the oil spill clean-up purpose. In this connection, it is necessary to develop nextgeneration adsorbent materials which can not only be easily synthesized but are also cost effective. There are recent reports on a few metal-organic frameworks (MOFs) that can be used for the oil spill cleanup purpose. ${ }^{33,34}$ However, a recent survey suggests that such MOF materials are not stable enough in the presence of water or moisture. ${ }^{35}$ So, the instability factor of MOFs transmits a shadow over their application in oil spill cleanup. Moreover, there is a high probability of leaching of metal ions by the hydration of MOFs, which may affect the water quality by intoxicating the aquatic system.

Herein, the facile synthesis of a melamine-based covalent organic polymer (CPCMERI-2) and its composite has been reported. The as-synthesized polymer has been thoroughly characterized by sophisticated solid-state analytical instrumentations like Fourier transform-IR and ${ }^{13} \mathrm{C}$ NMR to confirm the formation of the desired polymeric product. The hydrophobicity of the covalent polymer has been characterized by the measurement of contact angle, and the plausible cause for the hydrophobicity is well explained by AFM study. The growth study as well as the observation of the morphology of the grown COP has been done by FESEM analysis. The spilled oil clean-up experimentation has been carried out in ambient conditions and counter confirmed by IR spectroscopy.

\section{Experimental}

\section{Materials}

All chemicals used for the synthesis were of analytical grade. Melamine and isophthalaldehyde were purchased from SigmaAldrich and used without any further purification. Methanol and dimethylsulphoxide were obtained from Merck India. Kerosene and diesel were obtained from Durgapur steel plant. Solvents such as dichloromethane (DCM), tetrahydrofuran (THF), acetone, hexane (HXN), ethanol, benzene, nitrobenzene, toluene, chloroform and HPLC acetone were purchased from Merck India Pvt. Ltd. and used as obtained.

\section{Instrumentation}

FT-IR spectrometer (spectrum 65, Perkin Elmer) was used to obtain the FT-IR spectra (using $\mathrm{KBr}$ pellets). ${ }^{13} \mathrm{C}$ crosspolarisation solid-state NMR was performed using JEOL Resonance (Model ECX400) NMR spectrometer. Powder X-ray diffraction was performed using Bruker AXS (Model D8 Focus), Germany. The thermal stability of the synthesized covalent polymer, CPCMERI-2 was analyzed by NETZSCH STA 449F1 Jupiter in $\mathrm{Al}_{2} \mathrm{O}_{3}$ crucible in nitrogen atmosphere. A Quantachrome $\mathrm{iQ}_{2}$ surface area analyzer was used to measure nitrogen adsorption-desorption isotherms of the covalent polymer, CPCMERI-2, at 77 K. The surface morphology (FESEM) was investigated by sigma HD, Zeiss, Oxford Instruments, Germany. The surface profile was analysed by atomic force microscopy (AFM) using alpha 300 RAS, WITECH instruments. The contact angle measurements were performed by OCA 15 pro commercial goniometer (Dataphysics, Germany) equipped with a stepper motor for controlling the volume of the water dispensed from a microsyringe, and the digital images of the water droplets on the surface were captured by a CCD camera attached with the instruments in static mode.

\section{Synthesis of polyaminal network}

Melamine ( $1 \mathrm{mmol}$ ) was mixed in a $25 \mathrm{~mL}$ glass beaker with $10 \mathrm{~mL}$ of DMSO. The solution was transparent. After the 
solution became homogeneous, isophthalaldehyde $(1 \mathrm{mmol})$ was added. Instantly, the colour of the solution changed to pale yellow and remained unaltered up to its full dissolution. The homogenous solution was placed in a Teflon-lined autoclave and treated in a preheated hot air oven at $120^{\circ} \mathrm{C}$. After $72 \mathrm{~h}$ of reaction, the polymer was collected by filtration and washed several times thoroughly with acetone, DCM, THF, hexane etc. and soaked overnight in HPLC-grade acetone. Further purification was done using the soxhlet extraction process for 48 hours with methanol. The polymer was collected and left to air dry at $120{ }^{\circ} \mathrm{C}$ for 10 hours.

\section{Results and discussion}

COPs are considered as new-generation porous materials that are constructed by the tailor-made stitching of suitable organic monomers through rigid covalent bonding. In general, taking the advantage of designing by controllable modification, COPs are usually formed by the cross-linking of a number of organic building blocks bearing various functionalities. However, the use of low-cost precursors and a facile route for the fabrication of this type of polymer is always preferable in comparison to the most commonly used monomers with metal-based catalysts in high-temperature reaction conditions. Melamine, a costeffective and abundant chemical and a trimer of cyanamide that possesses a rigid 1,3,5-triazine skeletal moiety with three reactive primary amine groups, is recognized as one of the ideal precursors for the synthesis of organic polymers. In the present case, to verify the yield of the synthesized COP, the stoichiometry of melamine and isophthalaldehyde has been kept in three different levels. Table 1 represents the yield percent of the synthesized CPCMERI-2 using different molar ratios of the precursors at a reaction temperature of $120{ }^{\circ} \mathrm{C}$ in a Teflon-lined autoclave reactor. It has been observed that the stoichiometric ratio of aryl amine and melamine, herein, and aryl dialdehyde and isophthalaldehyde has an immense sway on the yield of the COP material. The chemical structure of the prepared melamine-based COP is shown in Scheme 1. TGA enlightens us about the thermal stability of CPCMERI-2 (Fig. S1, ESI $\dagger$ ). The chemical stability of the as-synthesized CPCMERI-2 has been checked, as reported in our previous work. ${ }^{36}$ The material displays good chemical stability in $2 \mathrm{~N} \mathrm{HCl}, 8 \mathrm{~N} \mathrm{NaOH}$ and even in Lewis acid-containing aqueous solutions.

\section{Structural analysis by FT-IR and ${ }^{13} \mathrm{C}-\mathrm{NMR}$}

The FT-IR spectrum of CPCMERI-2 using KBr pellet affirms the successful formation of the polymeric framework. Theoretically,

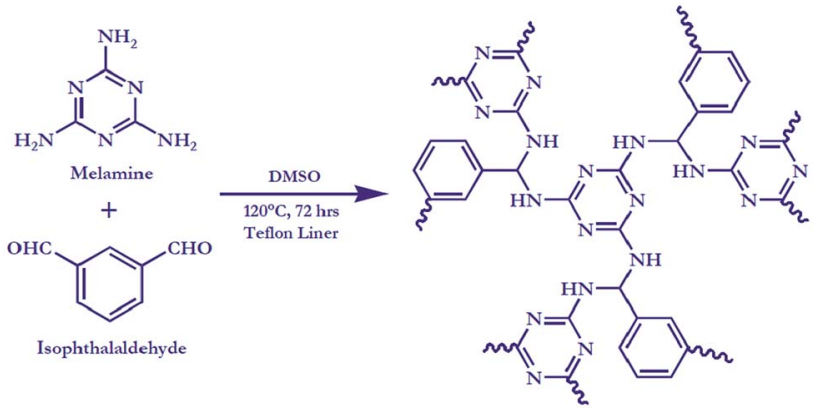

Scheme 1 Schematic representation of the synthesis of CPCMERI-2.

the condensation of melamine and isophthalaldehyde will produce two kinds of bonds, azomethine $(-\mathrm{C}=\mathrm{N}-)$ and aminal (-NH-C-NH-). However, vibrational stretching of the synthesized COP indicates the formation of an aminal linkage over azomethine despite the stoichiometry of the precursor materials. Bands appearing for $-\mathrm{NH}_{2}$ stretching and $-\mathrm{NH}_{2}$ deformation of the melamine unit at about $3470 \mathrm{~cm}^{-1}, 3420 \mathrm{~cm}^{-1}$ and $1650 \mathrm{~cm}^{-1}$ are reduced drastically in case of CPCMERI-2. The resonance of the $-\mathrm{C}=\mathrm{O}$ group at $1690 \mathrm{~cm}^{-1}$ and the $\mathrm{C}-\mathrm{H}$ stretching at $2870 \mathrm{~cm}^{-1}$ of isophthalaldehyde are absent in the spectrum of the covalent organic polymer. Distinct bands appear for quadrant stretching at $1545 \mathrm{~cm}^{-1}$ and for semicircle stretching at $1475 \mathrm{~cm}^{-1}$ of the triazine moiety, which implies the successful incorporation of melamine into the polymer. Moreover, the lack of azomethine $(\mathrm{C}=\mathrm{N}$ stretching) resonance around $1600 \mathrm{~cm}^{-1}$ confirms the formation of aminal bond in the provided reaction condition. Furthermore, a wide band that appears at $3315 \mathrm{~cm}^{-1}$ confirms the $\mathrm{N}-\mathrm{H}$ stretching of a secondary amine which is formed due to the condensation of the monomers particularly in aminal fashion as reported in some recently published articles. ${ }^{37-39}$ The single band that appears at $1335 \mathrm{~cm}^{-1}$ corresponds to the aromatic $\mathrm{C}-\mathrm{N}$ stretching of melamine (Fig. 1a). ${ }^{40}$

The formation of the polymeric framework of CPCMERI-2 has been further confirmed by solid-state cross-polarisation ${ }^{13} \mathrm{C}$ NMR spectroscopy. In the NMR spectrum of the polymer, the peak at about $\delta$ value 166.5 ppm appears for the carbon atoms present in alternate positions to the nitrogen atom in the triazine ring of melamine. The carbon responses at $\delta$ value of $130 \mathrm{ppm}$ and $135.6 \mathrm{ppm}$ correlate to the aromatic carbons of the benzene ring of the isophthalaldehyde unit. Peak appearing at $\delta$ value $60.6 \mathrm{ppm}$ corresponds to the carbon atoms present in the aminal linkage, as a result of the reaction between the amine and aldehyde moieties (Fig. 1b). No resonance is

Table 1 Stoichiometric ratios of precursors for the preparation of CPCMERI- 2 with the corresponding yield

\begin{tabular}{lll}
\hline Sl. no. & $\begin{array}{l}\text { Stoichiometry } \\
\text { (melamine : isophthalaldehyde) }\end{array}$ & Reaction condition \\
\hline 1 & $3: 2$ & $120^{\circ} \mathrm{C}$, Teflon liner \\
2 & $2: 3$ & $120^{\circ} \mathrm{C}$, Teflon liner \\
3 & $1: 1$ & $120^{\circ} \mathrm{C}$, Teflon liner
\end{tabular}




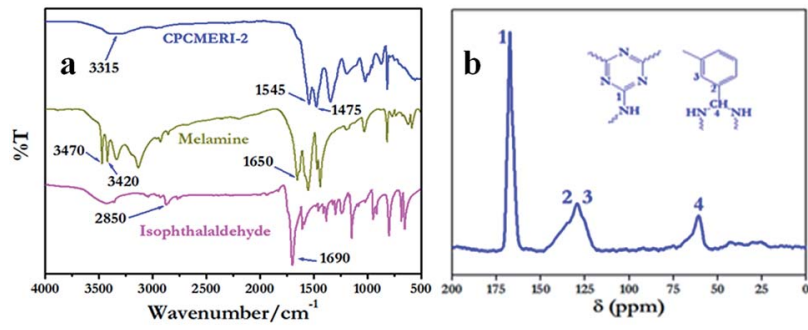

Fig. 1 FT-IR spectra (a) comparative spectra of CPCMERI-2, melamine and isophthalaldehyde; (b) solid-state cross-polarisation ${ }^{13} \mathrm{C} N \mathrm{NR}$ of the as-synthesized CPCMERI-2.

observed for the azomethine linkage $(\mathrm{C}=\mathrm{N})$ at $160 \mathrm{ppm}$, indicating the absence of an imine bond in the polymer networks; whereas, the disappearance of the resonance peak at $194 \mathrm{ppm}$ reflects the absence of unreacted aldehyde groups in the product, which is also confirmed by FTIR analysis. ${ }^{37-39}$

\section{PXRD analysis and measurement of the porous nature by surface analyser}

The wide-angle powder X-ray diffraction pattern is shown in Fig. 2a. The covalent polymer shows strong diffraction peaks with $2 \theta$ values of $21.76^{\circ}$ and $41.18^{\circ}$ along with a very weak intensity peak with $2 \theta$ value of $11.87^{\circ}$. The corresponding $d$ spacing values are $0.45 \mathrm{~nm}, 0.25 \mathrm{~nm}$, and $0.82 \mathrm{~nm}$, respectively. In the wide-angle powder-XRD analysis, before and after soxhlet purification, CPCMERI-2 shows a similar powder diffraction pattern indicating that the polymer is quite robust in nature even after a prolonged exposure in different solvent environments. The wide-angle PXRD analysis clearly indicates the amorphous nature of the polymer. It seems that the formation of the stacked layer structure by the ordered connection among the building blocks is otherwise difficult, suggesting the kinetically controlled synthetic process. ${ }^{41}$

\section{Sorption study of CPCMERI-2}

The nitrogen sorption isotherm of CPCMERI-2 was measured at $77 \mathrm{~K}$. CPCMERI-2 shows a typical type-I sorption isotherm profile without any significant hysteresis in the desorption branch of the isotherm (Fig. 2b). This reversibility in isotherms proves that the pore structure of CPCMERI-2 will not undergo deformation during the course of measurement of surface area
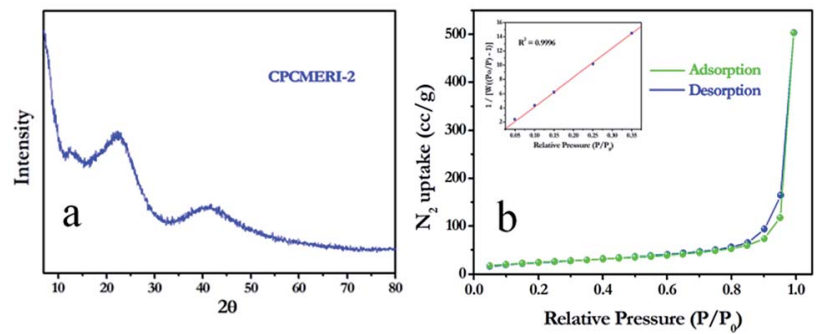

Fig. 2 (a) PXRD pattern of the as-synthesized CPCMERI-2; (b) $\mathrm{N}_{2}$ sorption isotherm of the as-synthesized CPCMERI-2. in liquid nitrogen; whereas, this confirms that the newly formed aminal bonds and the structural entities of CPCMERI-2 are rigid enough. ${ }^{42}$ The Brunauer-Emmett-Teller (BET) surface area of CPCMERI-2 is found to be $86.147 \mathrm{~m}^{2} \mathrm{~g}^{-1}$ and the Langmuir surface area is $147.73 \mathrm{~m}^{2} \mathrm{~g}^{-1}$. The cause of the low surface area of the polymer may be attributed to the lower temperature and humid condition of formation and the kinetically controlled reaction which increases the unordered connections between the building units. ${ }^{41}$ The average pore radius has been found to be in the range of $78.741 \AA$ i.e., exhibiting a pore diameter of $\sim 15.6 \mathrm{~nm}$ (Fig. S2, ESI $\uparrow$ ) with an average pore volume of $\sim 0.778$ $\mathrm{cm}^{3} \mathrm{~g}^{-1}$, reflecting the mesoporous nature of CPCMERI-2.

Owing to the presence of a basic secondary amine (-NH) group, CPCMERI-2 may have an affinity towards interaction with gases having an acidic character. $\mathrm{CO}_{2}$ is the commonest gas with Lewis acidic character and one of the vital members of the green house gases that is responsible for ozone layer depletion. Therefore, the use of a porous material to adsorb $\mathrm{CO}_{2}$ at ambient condition is required for the sake of our environment. To evaluate the $\mathrm{CO}_{2}$ uptake capacity at ambient condition, we have performed $\mathrm{CO}_{2}$ adsorption study at $298 \mathrm{~K}$ and up to 1 bar (normal atmospheric condition). CPCMERI-2 shows good uptake capability towards $\mathrm{CO}_{2}$. Even in the low-pressure region (0.3 bar) CPCMERI-2 can adsorb $\sim 11.5 \mathrm{~cm}^{3} \mathrm{~g}^{-1}$ of $\mathrm{CO}_{2}$. A sharp rise in the adsorption isotherm at the low-pressure region (up to 0.3 bar) indicates that CPCMERI-2 has good affinity towards $\mathrm{CO}_{2}$. CPCMERI-2 can uptake $20.7 \mathrm{~cm}^{3} \mathrm{~g}^{-1}$ or $38 \mathrm{mg}$ of $\mathrm{CO}_{2}$ under ambient condition $(298 \mathrm{~K}$ and 1 bar pressure) (Fig. S3, ESI $\dagger$ ).

\section{Wettability checking (hydrophobicity) and oil-sorption performance tests of CPCMERI-2}

A water droplet over the surface of CPCMERI-2 retains its spherical shape, and CPCMERI-2 renders the water droplet surprisingly nonwetting to the substrate, reflecting minimization of free energy at the water-air interface (Fig. 3a). The special compact structure of CPCMERI-2, because of its aromatic compact skeleton, makes it hydrophobic and thus the water droplet capable of rolling and retaining its spherical shape in this hydrophobic surface. The hydrophobicity of CPCMERI-2 has been ultimately investigated by contact angle measurement using the static mode. The contact angle of water

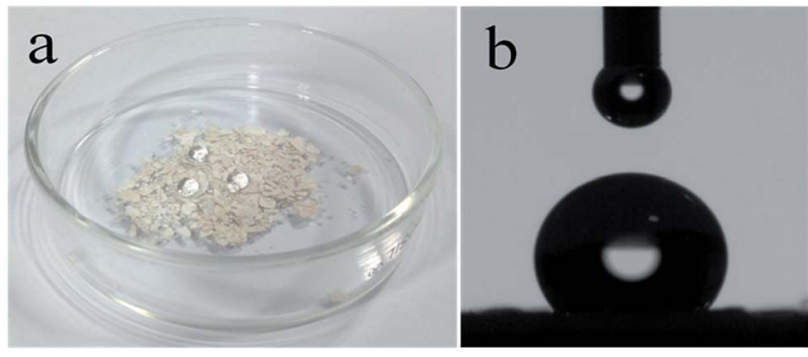

Fig. 3 (a) Water droplets (droplets volume $\sim 10 \mu \mathrm{L}$ ) stand still over CPCMERI-2, naked eye visualization, (b) contact angle of water droplet (droplet volume $\sim 10 \mu \mathrm{L}$ ) over CPCMERI-2-coated glass surface. 
droplets over CPCMERI-2-coated glass surface shows a high value of $130^{\circ}$ in a circle fitting mode (Fig. 3b), proving the hydrophobic nature of CPCMERI-2.

To gain further insight in the hydrophobic behaviour shown by CPCMERI-2, non-contact mode AFM imaging has been carried out using ethanol dispersion on CPCMERI-2-coated glass surface. 2D AFM imaging authenticates the presence of spherical particles, which is similar to the results shown in FESEM study (Fig. 4a and c). Those spherical particles exhibit needle-like protrusions in micro- and nano-scale regimes (Fig. 4b and d). Using 3D imaging mode for expanding into the nano region, the heights of the needle like extensions have been perceived to be in the range of 50-120 nm (Fig. 4d). The interspace region in between two needles plays a significant role for the material to execute its hydrophobic nature. The interspacing region is filled with trapped air generally known as air pockets which is responsible for the surface to be hydrophobic. ${ }^{43}$ Such type of uniform particle distribution throughout the surface generates a hills and valley type regime. According to the Cassie-Baxter model this type of surface is appropriate to produce hydrophobicity in a material. ${ }^{44-47}$

The hydrophobic nature as established by different sophisticated instrumentation techniques enthralled us towards the exploration of the oil-adsorption capability of the material over water surface. Indeed, incidents are known of fuel oil spillage over marine water which have caused a decrease in the oxygen level in water as well as a loss of fuel. ${ }^{47}$ In this regard, a robust hydrophobic material is one of the requirements to uptake fuel oils from over the water surface. The thermal and chemical resistance property, and the hydrophobic and oleophilic nature of CPCMERI-2 instigated us to check the performance of the developed polymeric framework towards the liquid-phase adsorption of fossil fuels like kerosene and diesel containing hydrocarbons of chain length $\mathrm{C} 10-\mathrm{C} 16$, which represent the major components of petroleum oil. ${ }^{48,49}$ Due to strong hydrophobicity and porosity, CPCMERI-2 readily adsorbs kerosene selectively over water surface as well as from solid surface without any significant difference in adsorption capacity.

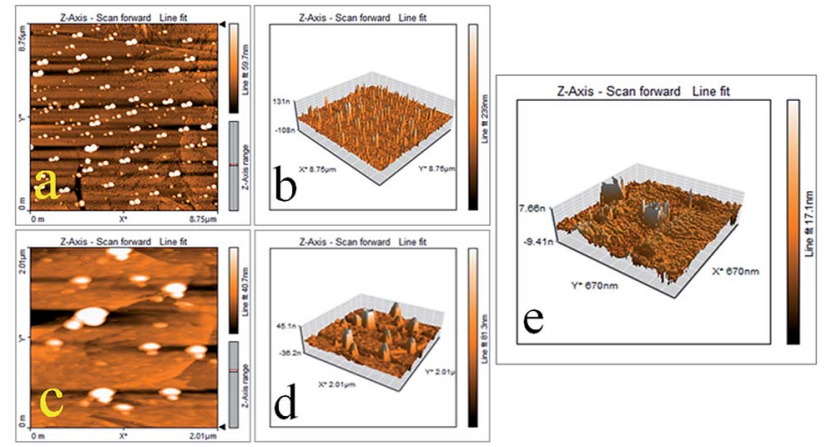

Fig. 4 AFM imaging of CPCMERI-2 microparticles-coated glass surface shows spherical agglomeration (a) 2D image in micro-region (scale $8.75 \mu \mathrm{m}^{2}$ ), (b) corresponding 3D image of the region, (c) highmagnification 2D image (scale $2.01 \mu \mathrm{m}^{2}$ ), (d) corresponding 3D image, and (e) nano-region magnification (scale $670 \mathrm{~nm}^{2}$ ) shows the rough surface over needle-like protrusions.
However, for other fuel substances and other organic solvents like benzene, toluene, xylene, nitrobenzene, and chloroform the uptake was substantially feeble. The uptake capacity of CPCMERI-2 for different fuel oils and organic solvents is tabulated in Table 2.

In this connection, for the real-day application, naturally abundant non-edible bio-waste materials together with CPCMERI-2 could be used for the adsorption of spilled oil over water surface. The peel of Citrus limetta (CL) has been utilized together with CPCMERI-2 for oil adsorption. In this relevance, for conducting oil adsorption studies, $200 \mathrm{mg}$ of CPCMERI-2 has been used in the form of pellets. Each pellet has been prepared by using a hydraulic pellet press applying 5 tons or $62.5 \mathrm{~kg} \mathrm{~cm} \mathrm{~cm}^{-2}$ of pressure for 2 minutes. In case of a biocomposite, CPCMERI-2 and the peel of Citrus limetta have been used in varying ratios to make the pellets in order to investigate the spilled oil adsorption ability. The adsorption capacity of each pellet has been thoroughly examined and concomitantly standardized after rigorous experimentations. A tactful experimentation by the proper permutation and combination of the ratio of CPCMERI-2 and the peel of Citrus limetta (CL) has been carried out in the following ratios: $4: 1$ (composite A), $2: 1$ (composite B) and 1: 1 (composite $\mathrm{C}$ ). The kerosene adsorption capacity of CPCMERI-2 and CL and each composite prepared using both CPCMERI-2 and CL has been tabulated (Table 3). Fig. 5 shows the as-synthesized CPCMERI-2, peel of Citrus limetta (CL), 1 : 1 composite of CPCMERI-2: CL and adsorbent pellets that have been used for the kerosene sorption study.

One of the prime features on which the adsorption of particular oil depends is the selection of structural units of the polyaminal material. It has been observed that the alteration of the aldehyde moiety in CPCMERI-2 leads to a change in oil uptake. Selectivity in oil adsorption is highly dependent on the reacting aldehyde. ${ }^{50}$

\section{FT-IR characterization of the as-synthesized CPCMERI-2, peel of Citrus limetta and guest analyte}

Kerosene is a mixture of long chain alkyl hydrocarbons as well as aryl hydrocarbons; therefore, the adsorption could be explored by FT-IR spectroscopy. The spectrum of CPCMERI-2 after kerosene adsorption shows strong stretching at $2900 \mathrm{~cm}^{-1}$, which could be attributed to the $-\mathrm{CH}_{2}$ unit of kerosene. ${ }^{51}$ Moreover, fingerprint stretching of CPCMERI-2 gets broadened and the $\mathrm{N}-\mathrm{H}$ stretching frequency at $3315 \mathrm{~cm}^{-1}$ is shifted to $3395 \mathrm{~cm}^{-1}$ due to the interaction of the $\mathrm{N}-\mathrm{H}$ proton of CPCMERI-2 with the polar moieties present in kerosene (Fig. 6a). In this way, the adsorption of kerosene by the peel of Citrus limetta (CL) can be explained by IR spectroscopy. The FTIR spectrum of CL after kerosene adsorption reveals major peaks at $2855 \mathrm{~cm}^{-1}$ and $2925 \mathrm{~cm}^{-1}$ due to $-\mathrm{CH}_{2}$ and $-\mathrm{CH}_{3}$ units of kerosene, respectively. ${ }^{52}$ Moreover, during the interaction of kerosene with CL fingerprint, the peak of CL at $1065 \mathrm{~cm}^{-1}$, which is due to the presence of $\mathrm{C}-\mathrm{O}$ group, is slightly shifted to $1035 \mathrm{~cm}^{-1}$. It may be interpreted to be due to the interaction of the $\mathrm{C}-\mathrm{O}$ group with the polar moieties present in kerosene (Fig. 6b). 
Table 2 Sorption capacity of CPCMERI-2 towards different light hydrocarbons

\begin{tabular}{llll}
\hline Sl. no. & Adsorbate & Uptake capacity of CPCMERI-2 & Adsorption condition \\
\hline 1 & Kerosene & $6.60 \mathrm{~mL} \mathrm{~g}^{-1}\left( \pm 0.2 \mathrm{~mL} \mathrm{~g}^{-1}\right)$ & Ambient condition \\
2 & Diesel & $3.25 \mathrm{~mL} \mathrm{~g}^{-1}\left( \pm 0.2 \mathrm{~mL} \mathrm{~g}^{-1}\right)$ & Ambient condition \\
3 & Benzene & $3.50 \mathrm{~mL} \mathrm{~g}^{-1}\left( \pm 0.2 \mathrm{~mL} \mathrm{~g}^{-1}\right)$ & Ambient condition \\
4 & Toluene & $3.85 \mathrm{~mL} \mathrm{~g}^{-1}\left( \pm 0.2 \mathrm{~mL} \mathrm{~g}^{-1}\right)$ & Ambient condition \\
5 & Nitrobenzene & $3.20 \mathrm{~mL} \mathrm{~g}^{-1}\left( \pm 0.2 \mathrm{~mL} \mathrm{~g}^{-1}\right)$ & Ambient condition \\
6 & Xylene & $4.20 \mathrm{~mL} \mathrm{~g}^{-1}\left( \pm 0.2 \mathrm{~mL} \mathrm{~g}^{-1}\right)$ & Ambient condition \\
7 & Chloroform & $3.90 \mathrm{~mL} \mathrm{~g}^{-1}\left( \pm 0.2 \mathrm{~mL} \mathrm{~g}^{-1}\right)$ & Ambient condition
\end{tabular}

Powder XRD characterization of the as-synthesized CPCMERI2, peel of Citrus limetta and guest analyte

The PXRD of the composite formed due to the mixing of CPCMERI-2 and the peel of Citrus limetta is shown in Fig. 7a. In Fig. 7b, the slight change in the PXRD pattern of CPCMERI-2 after kerosene uptake can be noticed. The peak at $2 \theta$ value of $21.76^{\circ}$ has a little shift of $\sim 4^{\circ}$, which can be attributed to the weak interaction between kerosene and the COP material. The corresponding $d$-spacing values are $0.44 \mathrm{~nm}$ and $0.55 \mathrm{~nm}$, respectively. The decrease in $2 \theta$ value implies the increase in the intersegmental distance of CPCMERI-2 due to interaction of CPCMERI-2 with kerosene. ${ }^{53}$ The slight shift in $2 \theta$ value supports the fact that interactive phenomenon occurring here is adsorption based and not an absorption type. ${ }^{54,55}$

\section{Morphological analysis by FESEM}

The nanomorphologies of CPCMERI-2, the peel of Citrus limetta and the composite material (CPCMERI-2 and bio-material) have been investigated using field-emission scanning electron microscopy (FESEM) and atomic force microscopy (AFM). FESEM study reveals that CPCMERI-2 is composed of agglomerated spherical particles with sizes ranging from $100 \mathrm{~nm}$ to $500 \mathrm{~nm}$ in diameter (Fig. 8a). This type of arrangement highlights the occurrence of micro-roughness on the surface of CPCMERI-2, and this particular feature explains the hydrophobicity of the material. FESEM analysis of the peel of Citrus limetta shows lopsided flakes-like morphology with some needle-like microstructures (Fig. 8c) with different sizes. The agglomeration of CPCMERI-2 in spherical form inspired us to evaluate the surface roughness through AFM.

\section{Recyclability study}

One step ahead, the recyclability of a sorbent is a very important characteristic for its practical application. In this relevance, CPCMERI-2 shows good recyclability towards kerosene uptake. Recyclability tests have been carried out successfully by allowing the material to soak kerosene up to saturation and recovering the kerosene from the COP by squeezing manually. The oil can be recovered easily by simply squeezing the polymer after adsorption of kerosene. The material can be reused four times during the successful removal of kerosene from water surface without any significant decrease in the amount of recovered kerosene. Fig. 9 shows the recyclability cycle of kerosene uptake by CPCMERI-2.

For the purpose of recyclability study of kerosene adsorption, $150 \mathrm{mg}$ of CPCMERI-2 is packed as a bed in a polypropylene column. After preparation of the adsorbent bed by CPCMERI-2 the weight of the column is measured using a Mettler Toledo analytical balance. A premeasured volume of kerosene is dispensed over the as-prepared CPCMERI-2 bed, and the adsorbent bed soaks the kerosene continuously until it reaches saturation. On reaching saturation, the weight of the column is carefully measured again. The difference in weight gives a clear indication of the amount of adsorbed kerosene. After the adsorption, the recovery process of kerosene is carried out manually by squeezing the adsorbent bed using a polyethylene piston. After recovery of the kerosene from the adsorbent bed, the weight of the column is measured again to find out the

Table 3 Sorption capacity of different adsorbents used for kerosene adsorption study along with the varying compositions of the composite adsorbents

\begin{tabular}{|c|c|c|c|c|c|}
\hline \multirow[b]{2}{*}{ Adsorbent } & \multicolumn{2}{|c|}{$\begin{array}{l}\text { Quantity of adsorbent used in } \\
\text { making pellet for oil-adsorption } \\
\text { study }\end{array}$} & \multirow[b]{2}{*}{$\begin{array}{l}\text { Ratio (CPCMERI-2 : peel } \\
\text { of Citrus limetta) }\end{array}$} & \multirow[b]{2}{*}{$\begin{array}{l}\text { Adsorption capacity } \\
\left(\mathrm{mL} \mathrm{g}^{-1}\right)\end{array}$} & \multirow[b]{2}{*}{ Adsorption condition } \\
\hline & CPCMERI-2 (mg) & $\begin{array}{l}\text { Peel of Citrus } \\
\text { Limetta (mg) }\end{array}$ & & & \\
\hline CPCMERI-2 & 200 & - & - & $6.6( \pm 0.2)$ & Ambient condition \\
\hline CPCMERI-2 and peel of Citrus limetta & 200 & 50 & $4: 1$ (composite A) & $6.85( \pm 0.2)$ & Ambient condition \\
\hline CPCMERI-2 and peel of Citrus limetta & 200 & 100 & $2: 1$ (composite B) & $7.10( \pm 0.2)$ & Ambient condition \\
\hline CPCMERI-2 and peel of Citrus limetta & 200 & 200 & $1: 1$ (composite $\mathrm{C})$ & $7.60( \pm 0.2)$ & Ambient condition \\
\hline Peel of Citrus limetta & - & 200 & - & $1.0( \pm 0.2)$ & Ambient condition \\
\hline
\end{tabular}




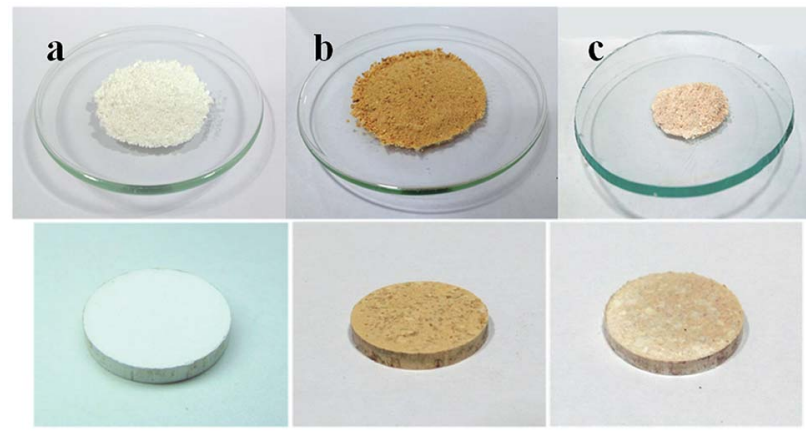

Fig. 5 Adsorbents used for kerosene adsorption study (a) as-synthesized CPCMERI-2, (b) peel of Citrus limetta (CL), and (c) $1: 1$ composite of CPCMERI-2 and peel of Citrus limetta. The bottom row shows the pellets prepared from the corresponding adsorbent materials.

amount of the recovered kerosene. Almost $70 \%$ of the adsorbed kerosene can be removed easily by simple squeezing. The remaining kerosene is removed by drying at $100{ }^{\circ} \mathrm{C}$. After drying, CPCMERI-2 is reused for the kerosene uptake study. Interestingly, it has been found that CPCMERI-2 can be used four times to salvage kerosene without hampering the percentage of recovered kerosene in each cycle. Moreover, after four rounds of the recycling study the polymer remains structurally robust although a trace amount of kerosene remains inside the polymer (Fig. S4, ESI $\dagger$ ). It is noticeable that although the uptake capacity of CPCMERI-2 is decreased during reuse of the material, the percentage of the recovered kerosene remains almost unaltered in every step of the recycling process. However, CPCMERI-2 experiences a decreased uptake of kerosene in the successive cycles. This decrease in the uptake capacity can be explained in terms of the active sites of the polymer available for interaction with kerosene. Kerosene consists of 36 organic compounds; among them, the maximum amount is of benzene, naphthalene and the analogues of these aromatic hydrocarbons. The cyclic aromatic hydrocarbons with higher boiling points may not be removed properly from the polymer during heat treatment in each step at $100{ }^{\circ} \mathrm{C}$ after the recovery of kerosene from the material via squeezing and in turn block the active sites of the polymer. The $\mathrm{CO}_{2}$ adsorption isotherms of CPCMERI-2 before and after recovering kerosene from the material prove the presence of some kerosene

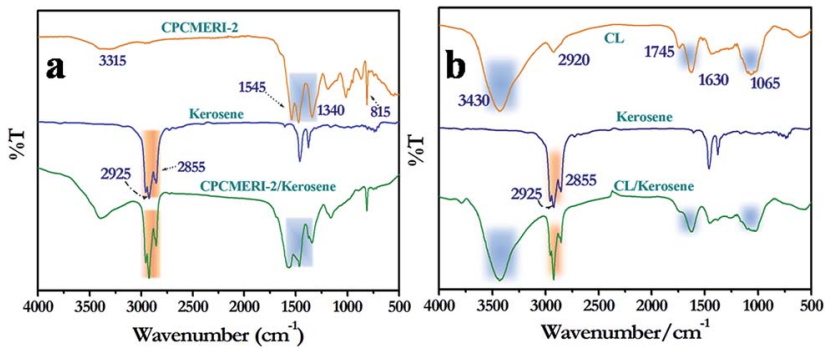

Fig. 6 FT-IR spectra (a) comparative spectra of CPCMERI-2, prior to adsorption, after adsorption of kerosene and kerosene; (b) spectra of $\mathrm{CL}$ prior to adsorption, after adsorption of kerosene, and kerosene.
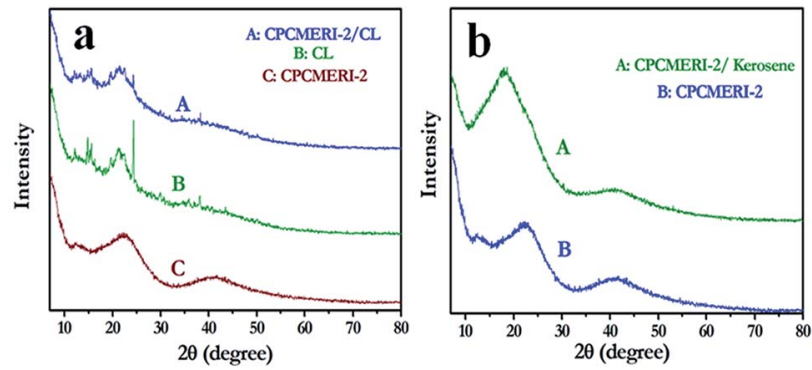

Fig. 7 PXRD of (a) comparative spectra of CPCMERI-2, CL and CPCMERI-2/CL composite prior to adsorption of kerosene; (b) spectra of CPCMERI-2 after adsorption of kerosene.

component in the polymer (Fig. S3, ESI $\dagger$ ). The as-synthesized CPCMERI-2 shows an uptake capacity of $20.7 \mathrm{~cm}^{3} \mathrm{~g}^{-1}$ of $\mathrm{CO}_{2}$; whereas, the material after kerosene adsorption can adsorb only $17.46 \mathrm{~cm}^{3} \mathrm{~g}^{-1}$ of $\mathrm{CO}_{2}$. The decrement in the adsorbed amount of $\mathrm{CO}_{2}$ is due to the fewer available active sites. Owing to this phenomenon, CPCMERI-2 experiences a decreased uptake in each successive cycle. However, the interaction of CPCMERI-2 with kerosene-like oils consisting of light hydrocarbons encourages us to develop a small-scale prototype for the oil spillage cleanup from wastewater, which is an essential step towards wastewater purification.

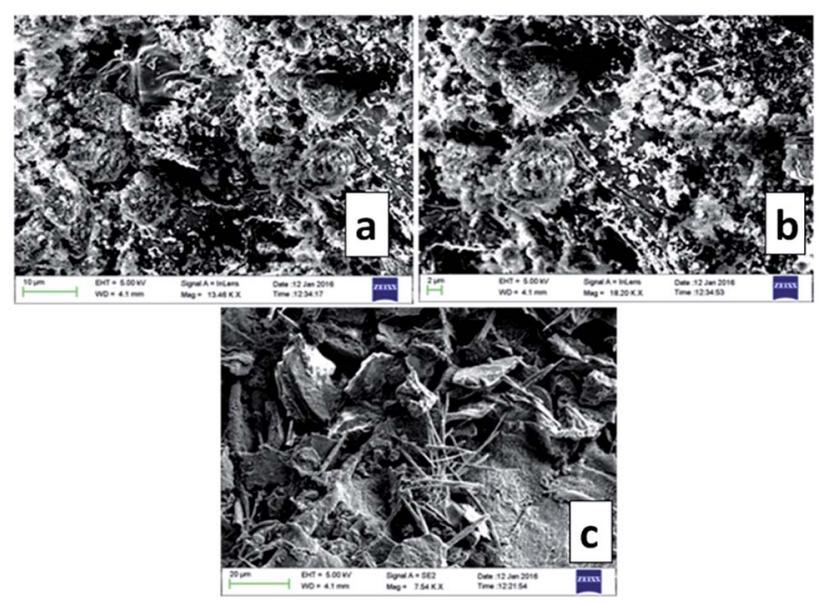

Fig. 8 FESEM analysis of (a) as-synthesized CPCMERI-2, (b) expanded view of spherical morphology, and (c) peel of Citrus limetta.

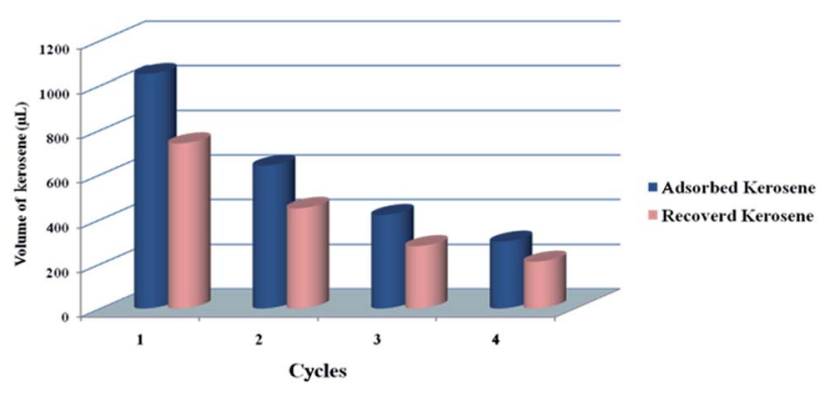

Fig. 9 Recyclability study of kerosene adsorption by CPCMERI-2. 


\section{Oil water separation: a precious approach for wastewater purification}

Wastewater purification is one of the major needs in today's perspective. Several adsorbent materials have been explored for removing water contaminants, but still there is a need for sorbent materials that can remove oil or oily particles from the water surface. Keeping this valuable point in our mind we have designed a small column-based filtration media which can easily separate kerosene/water mixture. Fig. 10 represents the filtration set-up used herein for the purpose of separation of kerosene/water mixture. The filtration setup contains a polypropylene barrel as the column. Inside the column $300 \mathrm{mg}$ of CPCMERI-2 is loaded by making a bed. The sorbent is supported by little amount of cotton so that the powder of CPCMERI-2 will not leach through the bottom nozzle of the column. Kerosene $(2 \mathrm{~mL})$ and industrial wastewater $(2 \mathrm{~mL})$ are taken in a beaker for the separation study. The wastewater has been procured from Durgapur Steel Plant and contains some carbon particles also. The steps in the purification process are marked stepwise as 1, 2, 3 and 4 in Fig. 10, and all the components of the purification setup are marked alphabetically from a to g. The collected industrial water already contains some amount of kerosene, but, additionally, $2.0 \mathrm{~mL}$ of kerosene

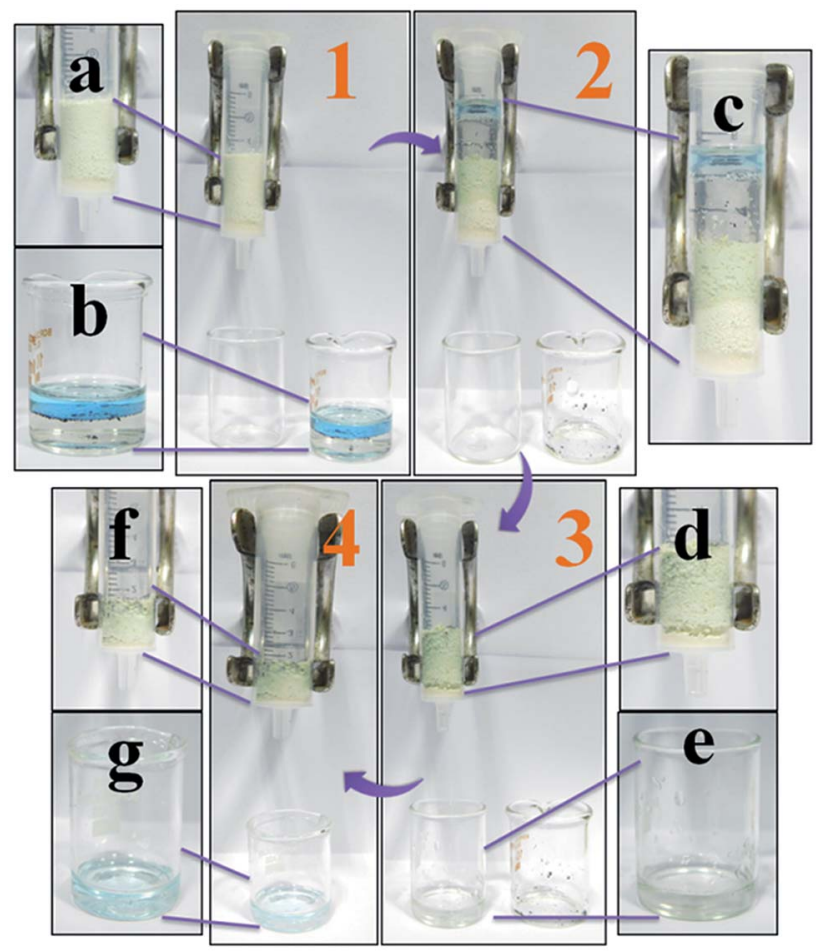

Fig. 10 Filtration setup for kerosene/wastewater mixture using CPCMERI-2. (1) CPCMERI-2 bed before pouring kerosene/wastewater mixture; [enlarged view: (a) CPCMERI-2 bed, (b) kerosene/industrial wastewater mixture], (2) kerosene/wastewater mixture over CPCMERI-2 bed; [enlarged view: (c) kerosene/wastewater mixture over CPCMERI-2 bed], (3) collection of filtered water; [enlarged view: (d) kerosene-adsorbed CPCMERI-2 bed, (e) filtered water], and (4) recovery of kerosene after water filtration; [enlarged view: (f) squeezed CPCMERI-2 bed, (g) collected kerosene]. is added to the water for better visualisation of the separation of kerosene, i.e., purification of water. In the first step, a mixture of kerosene and wastewater is poured over the packed bed of CPCMERI-2. The water in the column goes down slowly through the adsorbent bed, wherein the kerosene present in the mixture has been adsorbed by CPCMERI-2. During adsorption, the colour of the sorbent material is changed slightly, and is easily visible in Fig. 10. The filtrate water is collected in a beaker. In the next step, kerosene is recovered by manual pressing of CPCMERI-2 using a polypropylene piston. The recovered kerosene is collected in a separate beaker. The adsorbent can be reused for water purification by adsorbing kerosene from the water surface. This observation further motivated us to examine the performance of CPCMERI-2 towards kerosene adsorption over water surface.

\section{Cleanup of kerosene from water surface}

The as-synthesized covalent polymer (CPCMERI-2) could uptake kerosene floating over the water surface (vide Fig. 11 and $\mathrm{ESI}_{\dagger} \dagger$ ). Initially, water is taken in a Petridish and $2.0 \mathrm{~mL}$ of kerosene is added drop wise, which floats over the water surface making a thin layer of kerosene. Commercially available kerosene is blue in colour; therefore, it is clearly visible. Now, the covalent polymer is sprinkled on the kerosene floating over water. Within a minute CPCMERI-2 adsorbs the entire kerosene, thereby making the water clean (Fig. 11). Fig. 12 shows the covalent polymer when sprinkled on the water surface. Owing to its hydrophobicity, it does not get wet by water and eventually just floats on the water surface.

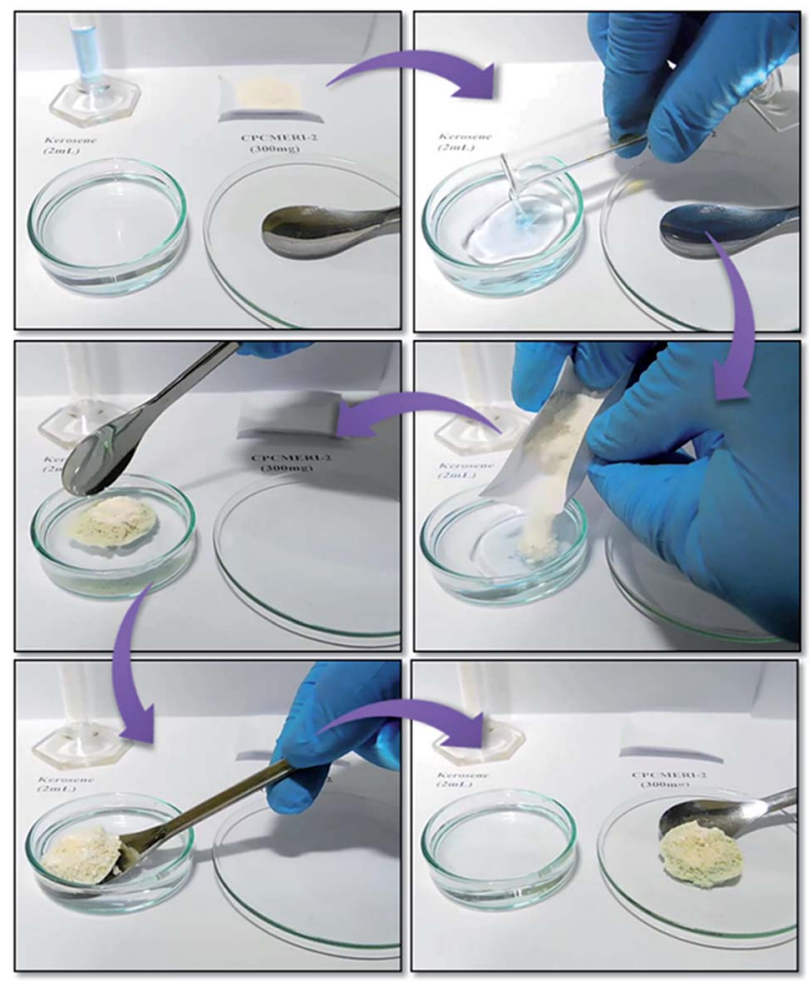

Fig. 11 Adsorption of kerosene by CPCMERI-2 over water surface. 


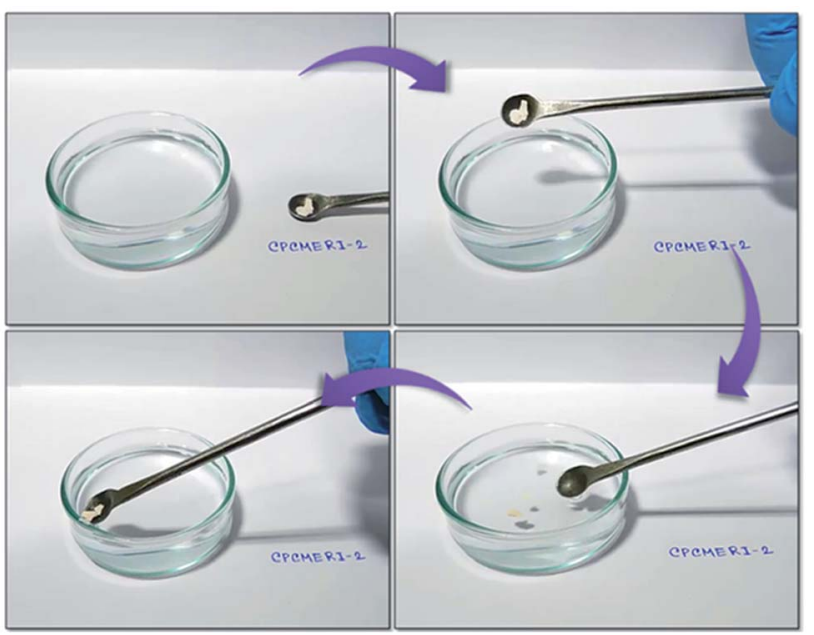

Fig. 12 Hydrophobic behaviour of CPCMERI-2 over water surface.

\section{Conclusions}

In summary, a melamine-based novel and multifunctional polyaminal material has been synthesized following a uni-step simple condensation process. The as-synthesized COP material has been well characterised by solid-state studies, e.g., IR, ${ }^{13} \mathrm{C}$ NMR, PXRD, and $\mathrm{N}_{2}$ sorption isotherm studies. The polyaminal material features hydrophobicity and oleophilicity with a static water contact angle of $130^{\circ}$. The observed hydrophobic nature of the as-developed melamine-based COP has been well described by AFM. Interestingly, the hydrophobicity and oleophilicity together make it a fascinating contender in separating kerosene-like petroleum oil fractions from contaminated water bodies. In consequence, the utilization of bio-waste materials like the peel of Citrus limetta as a composite material with the polyaminal COP material for the oil spill clean-up purpose increases the bio-compatibility as well as cost effectiveness of the as-synthesized material. This work leads to a new avenue and solid foundation for developing conjugated porous polymer materials as new types of sorbents for the removal of spilled oil residues from contaminated water and organic pollutants, and also has enormous potential in greenhouse gas storage, hydrocarbon like clean fuel storage and wastewater purification processes. The selective tuning of reactant components during the designed synthesis of the covalent polymer (CP) can lead to the selective adsorption of different petroleum fuels, which could have profuse impact in the recovery of mineral fuels from several wastewater bodies and its further use in automobile industries. This work asserts the emergence of such a COP material as a potential contender in developing a water resistant as well as chemically resistant hydrophobic material for producing a cleaner and greener environment.

\section{Conflicts of interest}

There are no conflicts to declare.

\section{Acknowledgements}

Council of Scientific \& Industrial Research (CSIR), Govt. of India, India (Ref. No. 33/2018/MD-FTT\&FTC and Project No. MLP-223712) is hereby acknowledged for financial support. The authors are thankful to SAIC of Tezpur University for PXRD facility and IISC Bangalore (SAIF) for NMR facilities. The authors are extremely thankful to Prof. Rahul Banerjee of IISER Kolkata for many helpful suggestions and thoughtful discussions. DD also gratefully acknowledges DST INSPIRE for his fellowship (INSPIRE Roll no: [IF160176]).

\section{Notes and references}

1 A. D. Mc Naught and A. Wilkinson, IUPAC Compendium of Chemical Terminology, 1997, p. 1598.

2 H. Schiff, Eur. J. Org. Chem., 1964, 131, 118-119.

3 H. Schiff, Justus Liebigs Ann. Chem., 1866, 140, 92-137.

4 R. P. Bisbey and W. R. Dichtel, ACS Cent. Sci., 2017, 3, 533543.

5 E. Vitaku and W. R. Dichtel, J. Am. Chem. Soc., 2017, 139, 12911-12914.

6 F. J. Uribe-romo, J. R. Hunt, H. Furukawa, C. Klo, M. O. Keeffe and O. M. Yaghi, J. Am. Chem. Soc., 2009, 131(13), 4570-4571.

7 A. K. Yudin and J. F. Hartwig, Catalyzed Carbon-Heteroatom formation, ISBN: 978-3-527-32428-6, Wiley-VCH, Weinheim, Germany, Oct 11, 2010.

8 X. Li, C. Zhang, S. Cai, X. Lei, V. Altoe, F. Hong, J. J. Urban, J. Ciston, E. M. Chan and Y. Liu, Nat. Commun., 2018, 9, 1-8.

9 Y. Yang, Q. Zhang, S. Zhang and S. Li, RSC Adv., 2014, 4, 5568-5574.

10 C. J. Doonan, D. J. Tranchemontagne, T. G. Glover, J. R. Hunt and O. M. Yaghi, Nat. Chem., 2010, 2, 235-238.

11 C. S. Diercks and O. M. Yaghi, Science, 2017, 355(6328), 923930.

12 D. B. Shinde, H. B. Aiyappa, M. Bhadra, B. P. Biswal, P. Wadge, S. Kandambeth, B. Garai, T. Kundu, S. Kurungot and R. Banerjee, J. Mater. Chem. A, 2016, 4, 2682-2690.

13 G. Mukherjee, J. Thote, H. B. Aiyappa, S. Kandambeth, S. Banerjee, K. Vanka and R. Banerjee, Chem. Commun., 2017, 53, 4461-4464.

14 P. D. Pathak, S. A. Mandavgane and B. D. Kulkarni, Curr. Sci., 2017, 113, 444-454.

15 S. Rafiq, R. Kaul, S. A. Sofi, N. Bashir, F. Nazir and G. Ahmad Nayik, J. Saudi Soc. Agric. Sci., 2018, 17(4), 351-358.

16 J. Michel and M. Fingas, Fossil Fuels, World Scientific Publishing Co., Inc., USA, 2016, ISBN: 978-981-4699-99-0.

17 The International Tanker Owners Pollution Federation Limited, Oil Tankers Spill Statistics, T. H. E. International, 2018.

18 C. A. Kontovas, H. N. Psaraftis and N. P. Ventikos, Mar. Pollut. Bull., 2010, 60, 1455-1466.

19 D. A. Edwards, R. G. Luthy and Z. Liu, Environ. Sci. Technol., 1991, 25, 127-133.

20 P. D. Sharma, Oil Spill Control, 2008, https:// saferenvironment.wordpress.com/tag/oil-spill-control/. 
21 L. Marsili, D. Coppola, N. Bianchi and S. Maltese, J. Environ. Anal. Toxicol., 2014, 5, 265.

22 O. E. Inoni, D. G. Omotor, F. N. Adun, O. E. Inoni, D. G. Omotor and F. N. Adun, J. Cent. Eur. Agr., 2006, 7, 41-48.

23 P. Thanikaivelan, N. T. Narayanan, B. K. Pradhan and P. M. Ajayan, Sci. Rep., 2012, 2, 230, DOI: 10.1038/srep00230.

24 Z. T. Li, B. Lin, L. W. Jiang, E. C. Lin, J. Chen, S. J. Zhang, Y. W. Tang, F. A. He and D. H. Li, Appl. Surf. Sci., 2018, 427, 56-64.

25 D. Wu, Z. Yu, W. Wu, L. Fang and H. Zhu, RSC Adv., 2014, 4, 53514-53519.

26 Z. R. Jiang, J. Ge, Y. X. Zhou, Z. U. Wang, D. Chen, S. H. Yu and H. L. Jiang, NPG Asia Mater., 2016, 8, 253-258.

27 G. Wang, B. Yu, S. Chen and H. Uyama, Sci. Rep., 2017, 7, 16.

28 Y. F. X. Chen, L. Liu, K. Liu and Q. Miao, J. Mater. Chem. A, 2014, 2, 10081-10089.

29 X. Sen Wang, J. Liu, J. M. Bonefont, D. Q. Yuan, P. K. Thallapally and S. Ma, Chem. Commun., 2013, 49, 1533-1535.

30 D. Liu, L. He, W. Lei, K. D. Klika, L. Kong and Y. Chen, Adv. Mater. Interfaces, 2015, 2, 1-6.

31 W. Lei, D. Portehault, D. Liu, S. Qin and Y. Chen, Nat. Commun., 2013, 4, 1777.

32 R. X. Yang, T. T. Wang and W. Q. Deng, Sci. Rep., 2015, 5, 1-9.

33 C. Yang, U. Kaipa, Q. Z. Mather, X. Wang, V. Nesterov, A. F. Venero and M. A. Omary, J. Am. Chem. Soc., 2011, 133, 18094-18097.

34 A. Banerjee, R. Gokhale, S. Bhatnagar, J. Jog, M. Bhardwaj, B. Lefez, B. Hannoyer and S. Ogale, J. Mater. Chem., 2012, 22, 19694-19699.

35 A. Carné-Sánchez, K. C. Stylianou, C. Carbonell, M. Naderi, I. Imaz and D. Maspoch, Adv. Mater., 2015, 27, 869-873.

36 D. Dey and P. Banerjee, New J. Chem., 2019, 43, 3769-3777.

37 M. G. Schwab, B. Fassbender, H. W. Spiess, A. Thomas, X. Feng and K. Mullen, J. Am. Chem. Soc., 2009, 131(21), 7216-7217.
38 K. Vellingiri, Y.-X. Deng, K.-H. Kim, J.-J. Jiang, T. Kim, J. Shang, W.-S. Ahn, D. Kukkar and D. W. Boukhvalov, ACS Appl. Mater. Interfaces, 2019, 11, 1426-1439.

39 A. Rehman and S. J. Park, Sci. Rep., 2018, 8, 1-11.

40 D. L. Pavia, G. M. Lampman, G. S. Kriz and J. R. Vyvyan, Introduction to Spectroscopy, Cengage Learning, 5th edn, 2015.

41 H. Ren, T. Ben, F. Sun, M. Guo, X. Jing, H. Ma, K. Cai, S. Qiu and G. Zhu, J. Mater. Chem., 2011, 21, 10348-10353.

42 G. Li, B. Zhang, J. Yan and Z. Wang, Chem. Commun., 2016, 52, 1143-1146.

43 Y. Wu, S. Jia, Y. Qing, S. Luo and M. Liu, J. Mater. Chem. A, 2016, 4, 14111-14121.

44 S. Roy, V. M. Suresh and T. K. Maji, Chem. Sci., 2016, 7(3), 2251-2256.

45 D. Mullangi, S. Shalini, S. Nandi, B. Choksi and R. Vaidhyanathan, J. Mater. Chem. A, 2017, 5(18), 8376-8384.

46 J. H. Hao and Z. J. Wang, J. Dispersion Sci. Technol., 2016, 37(8), 1208-1213.

47 A. Giacomello, S. Meloni, M. Chinappi and C. M. Casciola, Langmuir, 2012, 28(29), 10764-10772.

48 J. G. Speight, Handbook of Petroleum Product Analysis, Wiley, 2015, ISBN: 9781118986370.

49 J. G. Speight, Fuel Process. Technol., 1982, 5, 325-326.

50 P. Banerjee, P. Ghosh, D. Dey and N. C. Murmu, Patent under process in IPU, CSIR, New Delhi, 2017, Ref. No. 0083 NF2017.

51 N. L. Lam, K. R. Smith, A. Gauthier and M. N. Bates, J. Toxicol. Environ. Health, Part B, 2012, 15(6), 396-432.

52 M. Shakirullah, W. Ahmad, I. Ahmad, M. Ishaq and M. I. Khan, J. Chil. Chem. Soc., 2012, 57, 1375-1380.

53 G. Li, B. Zhang, J. Yan and Z. Wang, Macromolecules, 2014, 47(19), 6664-6670.

54 L. Pastero, D. Aquilano and M. Moret, Cryst. Growth Des., 2012, 12, 2306-2314.

55 C. X. Ren, L. X. Cai, C. Chen, B. Tan, Y. J. Zhang and J. Zhang, J. Mater. Chem. A, 2014, 2, 9015-9019. 